\title{
Musical training predicts cerebello-hippocampal coupling during music listening.
}

\section{Burunat, Ibella}

2018

Burunat , I , Brattico , E , Hartmann , M , Vuust , P , Särkämö , T \& Toiviainen , P 2018 , ' Musical training predicts cerebello-hippocampal coupling during music listening. ' , Psychomusicology, vol. 28 , no. 3 , pp. 152-163 . https://doi.org/10.1037/pmu0000215

http://hdl.handle.net/10138/311676

https://doi.org/10.1037/pmu0000215

acceptedVersion

Downloaded from Helda, University of Helsinki institutional repository.

This is an electronic reprint of the original article.

This reprint may differ from the original in pagination and typographic detail.

Please cite the original version. 
Running head: CEREBELLO-HIPPOCAMPAL COUPLING DURING LISTENING

Musical training predicts cerebello-hippocampal coupling during music listening Iballa Burunat

Finnish Centre for Interdisciplinary Music Research, Department of Music, Art and Culture Studies, University of Jyväskylä, Finland

Elvira Brattico

Center for Music in the Brain (MIB), Department of Clinical Medicine, Aarhus University \& The Royal Academy of Music Aarhus/Aalborg, Denmark

Martin Hartmann

Finnish Centre for Interdisciplinary Music Research, Department of Music, Art and Culture Studies, University of Jyväskylä, Finland

Peter Vuust

Center for Music in the Brain (MIB), Department of Clinical Medicine, Aarhus University \& The Royal Academy of Music Aarhus/Aalborg, Denmark Teppo Särkämö

Cognitive Brain Research Unit, Institute of Behavioural Sciences, University of Helsinki, Finland Petri Toiviainen

Finnish Centre for Interdisciplinary Music Research, Department of Music, Art and Culture Studies, University of Jyväskylä, Finland 


\begin{abstract}
Cerebello-hippocampal interactions occur during accurate spatio-temporal prediction of movements. In the context of music listening, differences in cerebello-hippocampal functional connectivity may result from differences in predictive listening accuracy. Using functional magnetic resonance imaging (fMRI), we studied differences in this network between 18 musicians and 18 nonmusicians while they listened to music. Musicians possess a predictive listening advantage over nonmusicians facilitated by strengthened coupling between produced and heard sounds through lifelong musical experience. Thus, we hypothesized musicians would exhibit greater functional connectivity than nonmusicians as a marker of accurate online predictions during music listening. To this end, we estimated the functional connectivity between cerebellum and hippocampus as modulated by a perceptual measure of the predictability of the music. Results revealed increased predictability-driven functional connectivity in this network in musicians compared to nonmusicians, which was positively correlated with the length of musical training. Findings may be explained by musicians' improved predictive listening accuracy. Our findings advance the understanding of cerebellar integrative function.
\end{abstract}

Keywords: fMRI, professional musicians, perception, plasticity, training 
Cerebellar function beyond the sensorimotor realm is becoming more widely accepted (Koziol et al., 2014; O’Reilly et al., 2010; Salmi et al., 2009; Salmi et al., 2010; Watson et al., 2015), evidencing an anterior sensorimotor versus posterior cognitive-emotional dichotomy in the cerebellum (Imamizu, Kuroda, Miyauchi, Yoshioka, \& Kawato, 2003; Stoodley, 2012; Koziol et al., 2014). Evidence gathered in the last twenty years supports cerebellar contributions to learning skills (Bellebaum and Daum, 2011), working memory and other language functions (Bellebaum and Daum, 2011; Leggio et al., 2011; Leiner, 2010; Schmahmann and Sherman, 1998; Steinlin, 2008; Tavano and Borgatti, 2010), spatial and episodic memory (Leggio et al., 2011; Rochefort et al., 2011; Schmahmann \& Sherman, 1998), emotion control (Colibazzi et al., 2010; Tavano \& Borgatti, 2010), event prediction (Forster \& Brown, 2011), empathy and predicting others' actions (Gazzola and Keysers, 2009; Ramnani and Miall, 2004; Schulte-Rüther et al., 2007; Singer et al., 2004), imitation (Jackson et al., 2006), planning and decision-making (Hogan et al., 2011; Ito, 2008; Tavano \& Borgatti, 2010), and cognitive developmental disorders including autism (Shukla et al., 2010; Steinlin, 2008). Notably, new research has reported important functional interactions between the posterior cerebellum and the hippocampus (Iglói et al., 2014; Krook-Magnuson et al., 2014; Onuki et al., 2015; Rochefort et al., 2011; Wikgren et al., 2010), for which several potential structural and functional connectivity pathways exist, evidenced by both animal and human studies. Animal studies have demonstrated direct connections between hippocampus and fastigial nucleus (Arrigo et al., 2014; Heath, Dempesy, Fontana, \& Myers, 1978; Robert G Heath \& Harper, 1974; Liu, Zhang, Yuan, Wang, \& Li, 2012; Oganesian, Melik-Musian, Fanardzhian, \& Grigorian, 1980; Snider \& Maiti, 1976; Wikgren et al., 2010; Yu, Gao, Wang, \& Chen, 1989). These findings support the existence of a direct anatomical substrate through which posterior cerebellum and hippocampus may influence one another. 
Thus, although no known path has been defined between the cerebellum and hippocampus, there is evidence for a bidirectional communication between these structures.

Moreover, a recent systematic review by Yu and Krook-Magnuson (Yu \& KrookMagnuson, 2015) on the novel area of cerebello-hippocampal (CER-HIPP) interactions emphasized the crucial role of CER-HIPP functional connectivity for spatial (Burguiere et al., 2005; Iglói et al., 2014; Petrosini, Leggio, \& Molinari, 1998; Rochefort et al., 2011; Rochefort, Lefort, \& Rondi-Reig, 2013) and temporal processing (Clark, Manns, \& Squire, 2002; Eichenbaum, 2014; Kirsch et al., 2003; Koekkoek et al., 2003; Logan \& Grafton, 1995; Paleja, Girard, Herdman, \& Christensen, 2014; Thompson \& Steinmetz, 2009; C. Weiss \& Disterhoft, 2011; Wikgren et al., 2010).

Using functional magnetic resonance imaging (fMRI), Onuki et al. (2015) observed coactivation between the left hippocampus and the cerebellum (bilateral lobule VI, right Crus I, left lobule VIIIb) during accurate spatio-temporal prediction of finger movements. More specifically, participants were prompted to press with one finger assigned buttons at a precise moment following visual cues (flashing moving markers). Thus, both temporal and spatial information were required for successfully predicting the precise moment and location of the finger press. Since CER-HIPP coupling was absent in conditions lacking the spatio-temporal integration component required to make accurate predictions (i.e., conditions requiring reactive instead of predicted finger movements, and an imagery version thereof), this coupling was thus interpreted to be an indicator of participants' accurate predictions based on integrating both spatial and temporal information.

In the present study we wanted to determine whether coupling between cerebellum and left hippocampus could be present during a perceptual condition that involves a predictive temporal component. Music listening provides an excellent context that relies 
heavily on predictive mechanisms without actual movement (Gebauer et al., 2015; Huron, 2006; Maidhof et al., 2010; Meyer, 1956; Narmour, 1990; Rohrmeier and Koelsch, 2012; Schenker, 1935; Schoenberg, 1978; Vuust et al., 2009). As experts in the musical domain, musicians possess optimized predictive models of musical structure allowing them to more accurately anticipate upcoming musical events (Drake \& Palmer, 2000; K. A. Ericsson \& Towne, 2010; Hansen, Vuust, \& Pearce, 2016; Lehmann \& Gruber, 2006). In addition, the superior abilities in timing and error correction observed in musicians have been attributed to the cerebellum (Chen, Penhune, \& Zatorre, 2008), a structure that, along with other motor-related areas, undergoes reorganization ostensibly by the impact of musical motor learning (Baer et al., 2015; Gaser \& Schlaug, 2003; Hutchinson, Lee, Gaab, \& Schlaug, 2003; Koeneke, Lutz, Wüstenberg, \& Jäncke, 2004; Ungerleider, Doyon, \& Karni, 2002). Because CER-HIPP coupling is suggested to be a marker of predictive accuracy (Onuki et al., 2015), differences in predictive listening accuracy may manifest as differences in CER-HIPP functional connectivity. Thus, a stronger CER-HIPP coupling in musicians compared to nonmusicians could be an indicator of improved predictive listening accuracy.

In the present study we used a functional magnetic resonance imaging (fMRI) paradigm that enables the use of naturalistic stimulation (continuous music) to study cognitive functions without the need for controlled tasks. We recorded fMRI brain responses from 18 musicians and 18 nonmusicians while they attentively listened to music of different genres. We were interested in CER-HIPP functional connectivity during moments of high predictability in the music (i.e., when participants are purportedly engaged in making accurate predictions).

The use of a perceptual segmentation task to obtain a measure of predictability in the current study was justified and motivated by background literature on information-theoretic 
descriptions of musical events as relating to their perceived predictability (cf. Juhász, 2004; Pearce, Müllensiefen, \& Wiggins, 2010).

The unpredictability (unexpectedness) of upcoming events is higher at event boundaries than elsewhere (Egermann et al., 2013; Hafer and Weiss, 1974; Harris, 1954; Juhász, 2004; Narmour, 1990; Pearce, Ruiz, Kapasi, Wiggins, \& Bhattacharya, 2010; Pearce and Wiggins, 2012; Shannon, 1951). In other words, segment boundaries are located at the peaks of highest information content of a signal (Abdallah \& Plumbley, 2009; Pearce \& Wiggins, 2006), Maximum-information-based segmentation methods have been similarly applied in text segmentation (Charniak, 2000; Reynar \& Ratnaparkhi, 1997; Ratnaparkhi, 1999; McCallum, Freitag, \& Pereira, 2000; Low, Ng, \& Guo, 2005), applying the information theory criterion as a marker of sentence boundaries. These boundaries mark the highest points of uncertainty in the signal because, after a segment boundary, next upcoming events are hardest to predict (less likely to be anticipated accurately).

This conforms with Event Segmentation Theory (EST; Zacks, Speer, Swallow, Braver, \& Reynolds, 2007), according to which an event boundary is distinguished when perceptual or conceptual features of the activity change, making the anticipation of upcoming information more difficult. At such points a transient increase in prediction error occurs, which gives rise to the subjective experience that a new event has begun (Zacks et al., 2007). Thus, measures of information content in the music can be obtained using segmentation tasks.

In the present study a real-time perceptual segmentation test was used to obtain segments in the music with high predictability (low information content). A different participant sample was used to avoid the effect of becoming familiar with the exposure. Participants had to identify segment boundaries defined as instants of significant change in 
the music. This allows us to measure the CER-HIPP connectivity during the segments of the music where prediction is possible since information content is low. Segment boundaries represent consequently an indirect but robust measure of predictability in the music based on information theory used recurrently in the literature.

The test was pooled separately for musicians and nonmusicians. The resulting boundaries identified in the music reflect the within-group consensual points of highest unpredictability in the music.

Using this segmentation approach, we are able to reliably obtain segments of the music where information content is low and thus accurate predictive listening is likely to happen. This variable was used to conduct psychophysiological interactions (PPI; Friston et al. 1997) analyses in order to estimate how the CER-HIPP functional connectivity depended on the predictability of the music. We hypothesized musicians to show increased predictability-driven functional connectivity in the CER-HIPP network compared to nonmusicians, as a marker of musicians' improved prediction accuracy during listening.

\section{Materials and Methods}

We proceeded as follows: first we obtained fMRI responses from participants during music listening. Following this, a perceptual test was conducted in a different participant pool (see Participants) to estimate the points of highest predictability in the music. Next, PPI analyses were performed between two hippocampal seeds and the cerebellum using the predictability variable. This was followed by t-tests between groups to find whether CER-HIPP coupling was driven by musical predictability differently in musicians and nonmusicians. Lastly, we investigated the relationship between musical training and predictability-driven functional connectivity in musicians.

\section{Participants}


FMRI experiment. 36 healthy participants with no history of neurological or psychological disorders participated in the fMRI experiment. The participants were screened for inclusion criteria before admission to the experiment (no ferromagnetic material in their body; no tattoo or recent permanent colouring; no pregnancy or breastfeeding; no chronic pharmacological medication; no claustrophobia) and upon admission to the experiment signed an informed written consent. The participant pool was selected to be equally divided between professional musicians $(\mathrm{n}=18$, age $=28.2 \pm 7.8$, females $=9)$ and nonmusicians $(\mathrm{n}=18$, age $=29.2 \pm 10.7$, females $=10$, left-handers $=1)$. The criteria for musicianship was having more than 5 years of music training, having finished a music degree in a music academy, reporting themselves as musicians, and working professionally as a performer. As for the type of musicians, there were classical $(n=12)$, jazz $(n=4)$, and pop $(n=2)$ musicians. The instruments played were strings (violin $=4$; cello $=2$; double bass $=1$ ), piano $(\mathrm{n}=8)$, winds (trombone $=1$; bassoon $=1)$, and mixed $(\mathrm{n}=1)$. The musicians' group was homogeneous in terms of the duration of their musical training, onset age of instrument practice, and amount of years of active instrument playing. These details were obtained and crosschecked via questionnaires and HIMAB (Gold et al. 2013; Helsinki Inventory for Music and Affect Behavior). There were no significant differences between the musician and nonmusician groups with respect to cognitive performance, socioeconomic status, or personality and mood questionnaire (see Supplementary Table 1 for a detailed list of background variables tested).

The experiment was undertaken with the understanding and written consent of all participants. The study protocol proceeded upon acceptance by the ethics committee of the Coordinating Board of the Helsinki and Uusimaa Hospital District. This study was part of a 
larger project (“Tunteet”) including several experimental sessions, fMRI paradigms, as well as questionnaires, and whose findings will be reported in separate papers.

Perceptual experiment. A separate participant pool $(\mathrm{N}=36)$ took part in the perceptual experiment (18 nonmusicians [7 females] and 18 musicians [10 females]). The rationale for using a different participant pool allows to minimize familiarity effects with the music which could affect the listening task during the fMRI scanning (or vice versa, the perceptual task) leading to participants reacting differently to cadential closure, repetition, and other features that could contribute to expectation violations. However, to minimize differences, groups were matched in terms of their demographic variables. The mean age of the participants was 27.45 years $(\mathrm{SD}=4.54)$. They were all students or graduates from different faculties of the University of Jyväskylä and of the JAMK University of Applied Sciences. Participants were rewarded with a movie ticket as a token for their participation. Musicians had an average of 14.39 years $(\mathrm{SD}=7.49)$ of musical training. The musical style played by 12 of the musicians was classical music, whereas the other 6 musicians played nonclassical musical styles. The main instruments played by participants were piano (5), guitar (4), flute (2), bass guitar, clarinet, saxophone, cello, violin, viola and voice. All the nonmusicians reported having had no musical training, whereas all of the selected musicians considered themselves either as semiprofessional (12) or professional (6 participants) musicians at the time of the data collection. None of the participants reported experience in dance, ballet or sound engineering. Six participants were very familiar with at least one stimulus but nobody reported having performed any of the examples. As a general rule, we referred to a participant as musician when he or she had reported more than 8 years of musical training and had also self-considered himself or herself as semiprofessional musician or professional musician. We discarded, for example, participants who, in a multiple-choice 
questionnaire, reported to be amateur musicians. In contrast, we considered participants to be nonmusicians if they considered themselves as nonmusicians and if they did not report any musical training.

\section{Stimuli}

Three musical pieces were used in the experiment: (a) Stream of Consciousness by Dream Theater; (b) Adios Nonino by Astor Piazzolla; and (c) Rite of Spring (comprising the first three episodes from Part I: Introduction, Augurs of Spring, and Ritual of Abduction) by Igor Stravinsky. These are a progressive rock/metal piece, an Argentinian New Tango, and an iconic 20th century classical work, respectively, thus covering distinct musical genres and styles. All three selected pieces are instrumental and have a duration of about 8 minutes (the recording details and Spotify links to the musical stimuli can be found in the Supplementary Document 1).

\section{FMRI Experimental Procedure}

Participants' brain responses were acquired while they listened to each of the musical stimuli in a counterbalanced order. For each participant the stimuli loudness was adjusted to a comfortable but audible level inside the scanner room (around $75 \mathrm{~dB}$ ). In the scanner, participants' only task was to attentively listen to the music delivered via high-quality MR-compatible insert earphones while keeping their eyes open.

\section{FMRI Scanning and Preprocessing}

Scanning was performed using a 3T MAGNETOM Skyra whole-body scanner (Siemens Healthcare, Erlangen, Germany) and a standard 20-channel head-neck coil, at the Advanced Magnetic Imaging (AMI) Centre (Aalto University, Espoo, Finland). Concurrent EEG was also acquired with BrainVision amplifier and the data will be reported elsewhere, not being of interest to the current study goal of fMRI signal reliability. Using a single-shot 
gradient echo planar imaging (EPI) sequence thirty-three oblique slices (field of view = 192x192 mm; 64x64 matrix; slice thickness $=4 \mathrm{~mm}$, interslice skip $=0 \mathrm{~mm}$; echo time $=32 \mathrm{~ms}$; flip angle $=75^{\circ}$ ) were acquired every 2 seconds, providing whole-brain coverage. T1-weighted structural images (176 slices; field of view $=256 \times 256 \mathrm{~mm}$; matrix $=256 \times 256$; slice thickness $=1 \mathrm{~mm}$; interslice skip $=0 \mathrm{~mm}$; pulse sequence $=$ MPRAGE) were also collected for individual coregistration. Functional MRI scans were preprocessed on a Matlab platform using SPM8 (Statistical Parametric Mapping), VBM5 for SPM (Voxel Based Morphometry; Ashburner and Friston, 2000); Wellcome Department of Imaging Neuroscience, London, UK), and customized scripts developed by the present authors. For each participant low-resolution images were realigned on six dimensions using rigid body transformations (translation and rotation corrections did not exceed $2 \mathrm{~mm}$ and $2^{\circ}$ respectively), segmented into grey matter, white matter, and cerebrospinal fluid, and registered to the corresponding segmented high-resolution T1-weighted structural images. These were in turn normalized to the MNI (Montreal Neurological Institute; Evans et al. 1994) segmented standard a priori tissue templates using a 12-parameter affine transformation. Functional images were then blurred to best accommodate anatomical and functional variations across participants as well as to enhance the signal-to-noise by means of spatial smoothing using an $8 \mathrm{~mm}$ full-width-at-half-maximum Gaussian filter. Movement-related variance components in fMRI time series resulting from residual motion artifacts, assessed by the six parameters of the rigid body transformation in the realignment stage, were regressed out from each voxel time series. Following this, spline interpolation was used to detrend the fMRI data, followed by temporal filtering (Gaussian smoothing with kernel width $=4 \mathrm{sec}$ ). 
We tested for differences in the amount of head movement between the groups by means of an independent samples t-test using participants' standard deviations of each of the 6 movement components, which resulted for any of the movement components in no significant differences at alpha $=0.05$.

Brain responses to the three stimuli were concatenated making a total of $\sim 24$ minutes worth of data. The rationale behind this was to combine stimuli representing a wide range of musical genres and styles in order to cancel out effects that the specific kinds of music may have on the phenomenon under investigation. The final time series had 702 samples after the 4 first samples of each of the three runs were removed to avoid artifacts due to magnetization effects.

\section{Hippocampal Seeds}

Guided by Onuki et al. (2015)'s findings, we used the left hippocampus as the seed for PPI analyses. Furthermore, due to the functional heterogeneity of the hippocampus, we divided the left hippocampus into anterior and posterior. The anterior hippocampus has been reported to be implicated in novelty processing, movement but also in stress, emotion and affect, while the posterior hippocampus seems to relate to familiarity, space-related processing, and performs primarily cognitive functions (Colombo et al., 1998; Fanselow and Dong, 2010; Strange et al., 1999).

We used an anatomical criterion for selecting two hippocampal seeds corresponding to anterior and posterior aspects of the left hippocampus. The uncus is a distinctive and recognizable landmark for parcellation of the hippocampus, which enables to distinguish the anterior (uncus) and posterior (body and tail) aspects of the hippocampus. Thus, the boundary between anterior and posterior hippocampal aspects was determined by the presence of the uncus in coronal slices. 
Following this, we created seeds by averaging the voxels time courses that fell into each of the anterior and posterior subareas of the left hippocampus. Using the Automated Anatomical Labeling (AAL; Tzourio-Mazoyer et al. 2002) mask, this happened at $\mathrm{Y}=55$.

\section{Perceptual Variable Representing Predictability}

We aimed to test whether CER-HIPP functional connectivity was modulated by the degree of predictability of the music more in musicians than in nonmusicians. PPI analyses served this purpose as they answer the question whether the strength of the functional connectivity depends on a third factor, in this case, a perceptual variable selected for representing the degree of predictability of the music. This variable was obtained in a real-time perceptual experiment, which took place with a computer in a sound-attenuated room. Participants were instructed in written form to mark instants of significant change as they listened to the music by pressing the space bar of the computer keyboard ("Your task is to mark instants of significant musical change by pressing the space bar of the computer keyboard. Whenever you find an instant of significant change, please press the spacebar key to mark it as you listen to the music. You will not have a chance to listen to the whole example before you start marking. Instead, during your first and only listen of each example, you will give us your 'first impression'”). After completing a trial, they listened and marked different musical stimuli, which were presented in a randomized order. Participants were instructed to give their "first impression" because they would not have a chance to listen to the whole example before they started marking. The interface included a play bar that offered basic visual-spatial cues regarding the beginning, current time position and end of the examples.

Each stimulus was presented to participants as four musical extracts:

- Piazzolla: 0-02:00, 01:57-03.57, 03:54-05:54, 05:51-08:07.968. 
- Dream Theater: 0-02:00, 01:57-03.57, 03:54-05:54, 05:51-07:50.979

- Stravinsky: 00:05-02:05, 02:02-04:02, 03:59-05:59, 05:56-07:52.243

We concatenated the segmentation data in order to obtain a set of indicated boundaries for the complete stimulus. The kernel density estimation (KDE; Silverman 1986) of these data was computed separately for musicians and nonmusicians to estimate its probability density curve. The chosen Gaussian kernel width was of 1.66 seconds, which was found to yield the optimal correlation between the KDE of musicians and nonmusicians. Between-group consistency was high $(r=.9, p<.001)$. However, nonmusicians seemed to indicate more segments in the music. The sampling interval used to compute the KDE was $10 \mathrm{~Hz}$. The KDE time series for each group was convolved with the canonical double-gamma hemodynamic response function (HRF) in order to match the hemodynamic response delay typical of blood-oxygen-level dependent (BOLD) brain responses, and downsampled to 0.5 $\mathrm{Hz}$ to match the sampling rate of the fMRI scanner.

The peaks of this curve were located where significant changes with highest consensus within groups occur. Predictability can be then derived from an information theory point of view. The degree of entropy or information content in the music would be maximal at the consensual boundaries, as these perceptual boundaries denote a significant change from preceding musical events and are thus not predictable from preceding musical cues. The same criterion has been previously used to detect segment boundaries in folk song melodies (Juhász, 2004), whereby high entropy implies a next interval hard to predict, at which point this may indicate a segment boundary. Thus, accurate predictive listening is likely to happen during segments of music between boundaries.

With the current segmentation approach, our predictability regressor describes the moments of highest unpredictability in the music (segment boundaries) with consistency 
across participants. Furthermore, the segmentation approach renders more reliable points of unpredictability in the music within the groups of interest, because it only focuses in the highest consensual points of unpredictability.

\section{Psychophysiological Interaction (PPI) Analyses}

This perceptual variable made it feasible to conduct PPI analyses to evaluate whether CER-HIPP functional connectivity was mediated by the degree of predictability of the music. PPI analyses are task-dependent functional connectivity analyses, which allow the study of how brain regions interact in a task-dependent manner (Friston et al., 1997). PPI measures how functional connectivity is affected by an external (psychological) variable, i.e., how the presence or absence of it modulates the functional connectivity. The statistical model for PPI is the multiple linear regression

$$
x_{i}=x_{k} \times g_{p} \cdot \beta_{i}+\left[x_{k} g_{p} G\right] \cdot \beta_{G}+e_{i}
$$

where $x_{k}$ denotes the physiological responses (the fMRI signal at a seed region, here the hippocampal seed), $g_{p}$ denotes the psychological variable (here the predictability of the music) convolved with a canonical hemodynamic response function (HRF), $x_{k} \times g_{p}$ represents the psychophysiological interaction term between the hippocampal seed activity and the predictability of the music, $x_{i}$ denotes the brain responses at each voxel within the cerebellum, $\beta_{i}$ denotes the beta parameter estimates corresponding to the PPI term; $\beta_{\mathrm{G}}$ is a matrix of the beta estimates corresponding to $x_{k}$ and $g_{p}$, as confounding variables, and other potential covariates of no interest $(\mathrm{G})$; and $e_{i}$ is the error term. Thus, the PPI term represents the explanatory variable in a multiple linear regression, and the inclusion of $x_{k}$ and $g_{p}$ as 
nuisance regressors guarantees any confounding effect induced by their variability alone to be ruled out. Cerebellar areas in which activity is best predicted by the PPI term indicate areas with strongest correlation with the hippocampal seed as a function of the predictability of the music.

The resulting beta parameter estimates were Z-transformed using the standard deviation of each of the beta distributions, calculated from the confidence intervals of the respective beta coefficients.

The significance of the Z-scores had to be estimated due to the intrinsic serial correlation of the fMRI time series derived from the smoothness of the hemodynamic response. To this purpose, we estimated the effective degrees of freedom $(d f)$ of the data following a nonparametric permutation-based approach (Pyper and Peterman, 1998) as shown in Eq. 2.

$$
\frac{1}{d f} \approx \frac{1}{N}+\frac{2}{N} \sum \frac{N-j}{N} \rho_{x x}(j) \rho_{y y}(j),
$$

where $\mathrm{N}$ is the number of observations, $\rho_{x}(j)$ and $\rho_{y y}(j)$ are the autocorrelations of the interaction term and a random cerebellar voxel time series at lag $j$, respectively. For each participant and hippocampal seed, the effective degrees of freedom were computed by randomly selecting 10,000 cerebellar voxels. Next, estimates from all trials across participants and seeds were averaged (mean $=306 \pm 5)$, and used to compute the significance of the Z-scores by dividing these by the standard error (see Eq. 3).

$$
z_{\text {corrected }}=z_{f} \sqrt{d f-3}
$$

\section{T-tests between Groups}

The Z-transformed PPI beta parameter estimates were compared between groups by means of t-tests (alpha $=0.01$, one-tailed). The choice of one-tailed t-tests responded to the 
need to test for directional differences between the groups. The resulting spatial maps were further corrected for multiple comparisons using a cluster-wise significance procedure based on permutation tests to derive a null distribution of the cluster sizes (CS) at a given significance level, from which a critical CS threshold can be selected at a particular FWE (family-wise error) rate. Specifically, group membership was bootstrap resampled with replacement and t-tests were performed at the alpha level given above. A critical cluster size of 60 voxels was obtained from a distribution of 10,000 cluster sizes $(\mathrm{FWE}=0.05)$.

Anatomical regions within each cluster were labeled based on the AAL (Automated Anatomical Labeling) atlas (Tzourio-Mazoyer et al., 2002) implemented in the MarsBaR toolbox v0.43 (http://marsbar.sourceforge.net). Clusters were also visually inspected using the probabilistic atlas of the human cerebellum implemented in FSL (https://fsl.fmrib.ox.ac.uk/fsl/fslwiki/) to ensure that the automatic assignment was conforming to the neurological knowledge. The $\mathrm{x} y \mathrm{z}$ coordinates (in MNI space) of the maximum voxel Z-value within each anatomical region were retrieved and accordingly labeled.

\section{Correlation Analyses with Years of Musical Training}

Additional correlation analyses tested a potential relationship between the duration of the musical training and the predictability-driven functional connectivity in musicians. Only significant voxels resulting from the group comparison were entered in the correlation analysis, i.e., those cerebellar areas with greater predictability-driven functional connectivity in musicians compared to nonmusicians. This would further support musical training as a driver for the increased CER-HIPP coupling during music listening.

Participants' Z-transformed beta coefficients of the respective cerebellar areas were correlated against the years of musical training across musicians. Spearman's rank correlation 
coefficient (alpha $=0.05$, one-tailed, uncorrected given the small voxel sets) was used since (a) the demographic variable was not normally distributed, and (b) its potential relationship with the PPI coefficients may not necessarily need to be a linear one. This non-parametric measure of dependence is in addition less sensitive to outliers.

\section{Results}

\section{T-tests between Groups}

Results from the t-tests $($ alpha $=0.01$, one-tailed; cluster-wise threshold $=60$ voxels, $\mathrm{FWE}=0.05)$ comparing the degree of modulation of the CER-HIPP functional connectivity by musical predictability (PPI analyses) yielded significantly greater predictability-driven functional connectivity in musicians for both hippocampal seeds compared to nonmusicians. Significant areas comprised the bilateral lobule VI and crus I (anterior seed), and the bilateral crus I-II, and right lobule VI (posterior seed). Effect sizes were also computed for all significant voxels. Large effect sizes (Cohen's d > 0.8) were extensively found $(71 \%$ and $100 \%$ of significant cerebellar voxels for the left anterior and left posterior hippocampal seeds, respectively), indicating that the difference between musicians' and nonmusicians' CER-HIPP functional connectivity is not only statistically significant but also substantially large. In other words, for both seeds musicians exhibited stronger CER-HIPP coupling than nonmusicians as the degree of musical predictability increased (see Figure 1 and Table 1 for list of regions).

[Figure 1 about here]

[Table 1 about here]

\section{Correlation Analyses with Years of Musical Training}

Correlation tests in musicians (Spearman, alpha $=0.05$ one-tailed) revealed significant results in the hypothesized direction of effect. This means that musicians with a longer 
musical training also exhibited stronger predictability-driven functional connectivity in the CER-HIPP network. Significant areas comprised right lobule VI and right crus I (anterior seed; see Table 2 for list of regions).

[Table 2 about here]

\section{Discussion}

We show here that the degree of predictability of the music had a significantly larger effect on musicians' CER-HIPP coupling compared to nonmusicians'. Additionally, the length of musical training was positively correlated with the degree of predictability-driven functional connectivity in musicians. In particular, our results revealed that musicians exhibited stronger CER-HIPP coupling than nonmusicians during segments of the music with low information content, where participants are more likely to predict upcoming musical events. The stronger CER-HIPP coupling could hence be a marker of more accurate predictive listening in musicians than in nonmusicians. We speculate action simulation to be a potential facilitating mechanism enabling accurate predictions. In other words, musicians, during listening, may be mentally simulating sound-producing actions. This simulation aids in generating predictions about subsequent musical events, a process facilitated via strengthened coupling between produced and heard sounds through life-long instrument practice.

Musical experience is crucially linked to prediction (Gebauer et al., 2015; Huron, 2006; Maidhof et al., 2010; Meyer, 1956; Narmour, 1990; Rohrmeier and Koelsch, 2012; Schenker, 1935; Schoenberg, 1978; Vuust et al., 2009), and musicians have been shown to exhibit stronger brain responses to expectation violations in musical contexts than nonmusicians (James et al., 2008; Koelsch et al., 2002; Koelsch et al., 2007; Oechslin et al., 2013; Vuust et al., 2011; Vuust et al., 2012). Previous research has determined that statistical 
learning produces information-theoretic descriptions of musical notes relative to their perceived expectedness, which additionally correspond to distinctive neural activity (Pearce et al., 2010). Furthermore, reinforcing this information-theoretic view, musicians have been shown to make better use of the predictive cues in low entropy contexts than controls to generate more accurate expectations, evidencing that musical training produces optimized predictive models of musical structure (Hansen et al., 2016; Hansen \& Pearce, 2014). In the same line, musicians outperform nonmusicians in making more successful online predictions about the forthcoming musical events given the current musical context (Mackay, 2003).

Our results conform with those by Onuki et al. (2015), who observed CER-HIPP functional connectivity only for accurate predictions. Musicians' stronger functional connectivity between cerebellum and hippocampus during moments of low information content in the music may reflect more accurate predictions being made by musicians compared to nonmusicians. This increased predictability-driven functional connectivity was observed in musicians between the cerebellum and both hippocampal seeds. Cerebellar areas comprised the bilateral lobule VI and crus I (anterior seed) and the bilateral crus I-II, and right lobule VI (posterior seed). These foci represent cognitive-related cerebellar regions in the posterior lobe, involved in higher-level tasks (spatial processing, executive functions, and

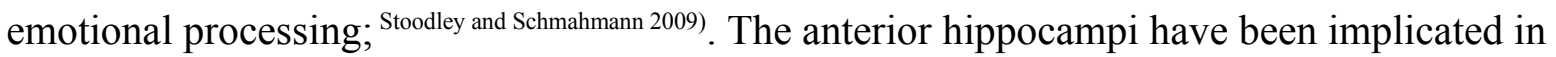
tasks involving novelty, movement, and emotion, in contrast with its posterior homologue, implicated in familiarity, space-related processing and cognition (Colombo et al., 1998; Fanselow and Dong, 2010; Strange et al., 1999). Accordingly, in light of what is known about hippocampal functional anteroposterior segregation, the greater implication of the anterior rather than the posterior hippocampi may highlight the novelty aspects of predictive processing during music listening. 
Furthermore, our present findings overlap with those by Onuki et al. (2015), who observed prediction-modulated functional connectivity between the left hippocampus and bilateral lobule VI, right Crus I, and left lobule VIII. In our study, the observed CER-HIPP coactivation extended also to the left Crus I and bilateral Crus II. Furthermore, all the cerebellar areas found (lobule VI and Crus I-II) are reached by a recently discovered anatomical pathway connecting hippocampus and cerebellum through the superior cerebellar penducle (Arrigo et al. 2014). Additionally, the cerebellar areas recruited are in line with the notion that a cognitive aspect, rather than a motor one, underlies the predictive component of the CER-HIPP coupling under investigation.

The positive correlation observed between years of musical training and predictability-driven CER-HIPP functional connectivity supported the role of musical training in driving the functional connectivity. Thus, cortico-subcortical reorganization seems to be influenced by the demands of musical training. Many studies have supported the assumption that the amount of musical training drives cortical plasticity (Gaser and Schlaug, 2003; James et al., 2014; Musacchia et al., 2007; Wong et al., 2007). For instance, individual variability in predicting upcoming tempo changes during a finger tapping task was positively correlated with amount of musical training (Pecenka and Keller, 2009). In our study, the CER-HIPP network showing the relationship between predictability-driven functional connectivity and years of musical training in musicians were lobule VI and crus I for the anterior seed. The involvement of the anterior hippocampus may emphasize aspects of novelty detection pertaining to the phenomenon of predictive processing of the musical structure. Moreover, cerebellar lobule VI is part of a neural mechanism mediating motor resonance (i.e., the activation of the motor system during action observation; Landmann et al. 
2011). In the context of music listening, this may support the hypothesis of action simulation performed by musicians during listening.

Moreover, previous research evidences the consistent involvement of the bilateral lobule VI in timing tasks that include a temporal-spatial perceptual prediction component (Keren-Happuch et al., 2014), which provides strong evidence supporting its role in musicians' predictive listening in the context of music perception. In sum, correlation analyses could further illuminate on the contribution of musical training to the modulation of the CER-HIPP network.

The present findings highlight the question of action simulation as the possible enhancing mechanism supporting the predictive listening ability in musicians. In musical contexts, action simulation is mediated by internal models that trigger auditory and motor images of one's own upcoming actions (Keller, 2008). Such action simulation would allow anticipating the future course of the perceived sounds (Wilson and Knoblich 2005; Sebanz and Knoblich 2009; Pezzulo et al. 2013). Since action simulation depends on the observer's own action experience (Bangert et al., 2006; Baumann et al., 2005; Lahav et al., 2007), it is thus particularly strong in professional musicians, given their lifelong experience-based associations between sensory and motor processes (Zatorre et al., 2007). In addition, action simulation mechanisms are more readily triggered during music listening in musicians than nonmusicians due to stronger coupled sensorimotor loops (Bangert et al., 2006; Gebel et al., 2013; Haslinger et al., 2005; Kajihara et al., 2013; Lotze et al., 2003; Schulz et al., 2003; Stewart et al., 2003; Zatorre et al., 2007). Consequently, musicians may be making accurate predictions during music listening to a greater extent than nonmusicians on the basis of action simulation mechanisms. 
Action simulation and internal models have been documented to be encoded in the cerebellum in connection with other brain regions, by forming and accessing internal models that facilitate predictions towards the desired goals of cognition in an error-free manner (Ito, 2008). The CER-HIPP loop may be one of the neural mechanisms acting as a facilitator for online spatio-temporal predictions.

\section{Limitations}

There are several limitations to the current methodology that should be noted. First, the musician samples used in the behavioral and fMRI experiments differ in the average level of musicianship, although no amateur musicians were used for either experiment. Second, although there are advantages to using different types of musicians to capture the general aspects of musicianship rather than the specificities of a target profile of musician, we acknowledge the disadvantage that concerns musicians playing monophonic (e.g., trombone) versus polyphonic (e.g., piano) instruments and their varying levels in prediction accuracy for polyphonic music. Third, participants (both fMRI and perceptual experiment pools) were asked to rate their familiarity with the stimuli on a scale from 1 to 5 . Musicians were overall more familiar with two of the three musical pieces used than nonmusicians (Adios Nonino and Rite of Spring), while there were no differences for Stream of Consciousness. However, potential musician-nonmusician differences in familiarity to the musical stimuli would be accordingly reflected in the predictability measure, which is a group-specific measure (see Perceptual Variable Representing Predictability). This would in turn account for betweengroup differences in familiarity to the stimuli. Furthermore, we argue that it may be challenging to disentangle schematic from veridical expectations (Justus \& Bharucha, 2001) on the neural level (i.e., automatic, learned-through-exposure expectations derived from music-syntactic rules [schematic expectations] vs expectations in a familiar musical piece 
[veridical expectations]). Evidence shows that musical training increases predictive accuracy during music listening (Mackay 2003; Hansen \& Pearce, 2014). Furthermore, musicians seem to possess schematic knowledge for music styles they are not familiar with for which they exhibit increased predictive accuracy compared to nonmusicians (Hansen et al., 2016). In addition, it has been shown that expectations based on listeners' schematic knowledge of music seem to resist veridical expectancies, evidencing the contribution of expectations despite listeners' familiarity about what will come next (Tillmann \& Bigand, 2010). Finally, it should be noted that the approach used does not provide any information regarding the directionality of information flow between cerebellum and hippocampus.

\section{Conclusion}

Previous research has suggested CER-HIPP functional connectivity as a marker of accurate spatio-temporal prediction of finger movements. The present study is the first to show CER-HIPP coupling in the absence of explicit movement, while participants listened attentively to music. We further established a relationship between the predictability of the music, participants' musical expertise, and CER-HIPP functional connectivity during music listening. Our findings overlap with those by Onuki et al. (2015) and provide novel evidence for increased CER-HIPP functional integration in musicians as a function of musical predictability compared to nonmusicians, lending further support to the hypothesis of musicians' functional consolidation (plasticity) as a result of their long-term musical training. Furthermore, the present study uses a paradigm that employs a task consisting only in listening attentively to continuous music. This setting provides increased ecological validity compared to previous approaches in the study of CER-HIPP interaction.

Our current results substantiate and extend previous findings on CER-HIPP coupling while aiding to elucidate the role of this functional network in the context of music listening. In 
addition, these findings advance the understanding of cerebellar integrative function by extending previous knowledge on cerebellar contributions in the context of prediction and emphasizing the cerebellar role in higher mental functions in healthy physiology. Because the cerebellum is compromised in several behavioral and cognitive developmental and degenerative disorders, as evidenced by neuropsychological, morphological and functional imaging studies (Bugalho et al., 2006; Peng et al., 2013; Phillips et al., 2015), the present results are also of clinical significance for disentangling and interpreting the different contributions of specific cerebellar areas in an integrative manner in pathological behavior and cognitive functioning.

\section{References}

Abdallah, S., \& Plumbley, M. (2009). Information dynamics: patterns of expectation and surprise in the perception of music. Connection Science, 21(2-3), 89-117.

Arrigo, A., Mormina, E., Anastasi, G. P., Gaeta, M., Calamuneri, A., Quartarone, A., ... Milardi, D. (2014). Constrained spherical deconvolution analysis of the limbic network in human, with emphasis on a direct cerebello-limbic pathway. Frontiers in Human Neuroscience, 8(December), 1-11. https://doi.org/10.3389/fnhum.2014.00987

Ashburner, J., \& Friston, K. J. (2000). Voxel-based morphometry--the methods. NeuroImage, 11(6 Pt 1), 805-21. https://doi.org/10.1006/nimg.2000.0582

Baer, L. H., Park, M. T. M., Bailey, J. a, Chakravarty, M. M., Li, K. Z. H., \& Penhune, V. B. (2015). Regional cerebellar volumes are related to early musical training and finger tapping performance. NeuroImage, 109, 130-9. https://doi.org/10.1016/j.neuroimage.2014.12.076

Bangert, M., Peschel, T., Schlaug, G., Rotte, M., Drescher, D., Hinrichs, H., ... Altenmüller, 
E. (2006). Shared networks for auditory and motor processing in professional pianists: evidence from fMRI conjunction. NeuroImage, 30(3), 917-26. https://doi.org/10.1016/j.neuroimage.2005.10.044

Baumann, S., Koeneke, S., Meyer, M., Lutz, K., \& Jäncke, L. (2005). A network for sensorymotor integration: what happens in the auditory cortex during piano playing without acoustic feedback? Annals of the New York Academy of Sciences, 1060, 186-8. https://doi.org/10.1196/annals.1360.038

Bellebaum, C., \& Daum, I. (2011). Mechanisms of cerebellar involvement in associative learning. Cortex, 47(1), 128-136.

https://doi.org/http://dx.doi.org/10.1016/j.cortex.2009.07.016

Bugalho, P., Correa, B., \& Viana-Baptista, M. (2006). Papel do cerebelo nas funções cognitivas e comportamentais. Bases Científicas e Modelos de Estudo [Role of the cerebellum in cognitive and behavioural control: scientific basis and investigation models]. Acta Medica Portuguesa, 19(3), 257-268. https://doi.org/17234089

Burguiere, E., Arleo, A., Hojjati, M. R,, Elgersma, Y., De Zeeuw, C. I., Berthoz, A., \& Rondi-Reig, L. (2005). Spatial navigation impairment in mice lacking cerebellar LTD: a motor adaptation deficit? Nat Neurosci, 8(10), 1292-1294.

Charniak, E. (2000). A maximum-entropy-inspired parser. In Proceedings of the 1st North American chapter of the Association for Computational Linguistics conference (pp. 132-139). Association for Computational Linguistics.

Chen, J., Penhune, V. B., \& Zatorre, R. J. (2008). Moving on time: Brain network for auditory-motor synchronization is modulated by rhythm complexity and musical training. Journal of Cognitive Neuroscience, 20(2), 226-239. 
Clark, R. E., Manns, J. R., \& Squire, L. R. (2002). Classical conditioning, awareness, and brain systems. Trends in Cognitive Sciences, 6(12), 524-531. https://doi.org/http://dx.doi.org/10.1016/S1364-6613(02)02041-7

Colibazzi, T., Posner, J., Wang, Z., Gorman, D., Gerber, A., Yu, S., ... Peterson, B. S. (2010). Neural systems subserving valence and arousal during the experience of induced emotions. Emotion. Colibazzi, Tiziano: College of Physicians and Surgeons and New York State Psychiatric Institute, Unit 74, 1051 Riverside Drive, New York, NY, US, 10032, tc2237@columbia.edu: American Psychological Association. https://doi.org/10.1037/a0018484

Colombo, M., Fernandez, T., Nakamura, K., \& Gross, C. G. (1998). Functional differentiation along the anterior-posterior axis of the hippocampus in monkeys. Journal of Neurophysiology, 80(2), 1002-1005.

Diedrichsen, J., Maderwald, S., Kueper, M., Thuerling, M., Rabe, K., Gizewski, E. R., ... Timmann, D. (2011). Imaging the deep cerebellar nuclei: A probabilistic atlas and normalization procedure. NeuroImage, 54(3), 1786-1794.

https://doi.org/10.1016/j.neuroimage.2010.10.035

Drake, C., \& Palmer, C. (2000). Skill acquisition in music performance: relations between planning and temporal control. Cognition, 74(1), 1-32. https://doi.org/http://dx.doi.org/10.1016/S0010-0277(99)00061-X

Duvernoy, H. M. (2005). The human hippocampus: functional anatomy, vascularization and serial sections with MRI. Springer Science \& Business Media.

Egermann, H., Pearce, M. T., Wiggins, G. a, \& McAdams, S. (2013). Probabilistic Models of Expectation Violation Predict Psychophysiological Emotional Responses to Live Concert Music. Cognitive, Affective \& Behavioral Neuroscience, 13(3), 533-553. 
https://doi.org/10.3758/s13415-013-0161-y

Eichenbaum, H. (2014). Time cells in the hippocampus: a new dimension for mapping memories. Nat Rev Neurosci, 15(11), 732-744.

Ericsson, K. A., \& Towne, T. J. (2010). Expertise. Wiley Interdisciplinary Reviews: Cognitive Science, 1(3), 404-416. https://doi.org/10.1002/wcs.47

Evans, A., Kamber, M., Collins, D., \& MacDonald, D. (1994). An MRI-based probabilistic atlas of neuroanatomy. In S. D. Shorvon (Ed.), Magnetic Resonance Scanning and Epilepsy (pp. 263-274). New York: Plenum Press.

Fanselow, M. S., \& Dong, H.-W. (2010). Are the dorsal and ventral hippocampus functionally distinct structures? Neuron, 65(1), 7-19. https://doi.org/10.1016/j.neuron.2009.11.031.Are

Forster, S. E., \& Brown, J. W. (2011). Medial Prefrontal Cortex Predicts and Evaluates the Timing of Action Outcomes. NeuroImage, 55(1), 253-265. https://doi.org/10.1016/j.neuroimage.2010.11.035

Friston, K. J., Buechel, C., Fink, G. R., Morris, J., Rolls, E., \& Dolan, R. J. (1997). Psychophysiological and modulatory interactions in neuroimaging. NeuroImage, 6(3), 218-29. https://doi.org/10.1006/nimg.1997.0291

Gaser, C., \& Schlaug, G. (2003). Brain Structures Differ between Musicians and NonMusicians, 23(27), 9240-9245.

Gazzola, V., \& Keysers, C. (2009). The Observation and Execution of Actions Share Motor and Somatosensory Voxels in all Tested Subjects: Single-Subject Analyses of Unsmoothed fMRI Data. Cerebral Cortex , 19(6), 1239-1255.

Gebauer, L., Kringelbach, M. L., \& Vuust, P. (2015). Predictive coding links perception, action, and learning to emotions in music. Comment on "The quartet theory of human 
emotions: An integrative and neurofunctional model" by S. Koelsch et al. Physics of Life Reviews, 1, 21-23. https://doi.org/10.1016/j.plrev.2015.04.023

Gebel, B., Braun, C., Kaza, E., Altenmüller, E., \& Lotze, M. (2013). Instrument specific brain activation in sensorimotor and auditory representation in musicians. NeuroImage, 74, 37-44. https://doi.org/10.1016/j.neuroimage.2013.02.021

Gloor, P. (1997). The temporal lobe and limbic systemOxford University Press. New York, 865.

Gold, B. P., Frank, M. J., Bogert, B., \& Brattico, E. (2013). Pleasurable music affects reinforcement learning according to the listener. Frontiers in Psychology, 4(August), 119. https://doi.org/10.3389/fpsyg.2013.00541

Hafer, M. A., \& Weiss, S. F. (1974). Word segmentation by letter successor varieties. Information Storage and Retrieval, 10(11), 371-385.

Hansen, N. C., \& Pearce, M. T. (2014). Predictive uncertainty in auditory sequence processing. Frontiers in Psychology, 5(SEP), 1-17. https://doi.org/10.3389/fpsyg.2014.01052

Hansen, N. C., Vuust, P., \& Pearce, M. (2016). "If You Have to Ask, You'll Never Know": Effects of Specialised Stylistic Expertise on Predictive Processing of Music., 1-20. https://doi.org/10.1371/journal.pone.0163584

Harris, Z. S. (1954). Distributional structure. Word, 10(2-3), 146-162. https://doi.org/10.1080/00437956.1954.11659520

Haslinger, B., Erhard, P., Altenmüller, E., Schroeder, U., Boecker, H., \& Ceballos-Baumann, a O. (2005). Transmodal sensorimotor networks during action observation in professional pianists. Journal of Cognitive Neuroscience, 17(2), 282-93. https://doi.org/ $10.1162 / 0898929053124893$ 
Heath, R. G., Dempesy, C. W., Fontana, C. J., \& Myers, W. A. (1978). Cerebellar stimulation: Effects on septal region, hippocampus, and amygdala of cats and rats. Biological Psychiatry. Netherlands: Elsevier Science.

Heath, R. G., \& Harper, J. W. (1974). Ascending projections of the cerebellar fastigial nucleus to the hippocampus, amygdala, and other temporal lobe sites: Evoked potential and histological studies in monkeys and cats. Experimental Neurology, 45(2), 268-287. https://doi.org/http://dx.doi.org/10.1016/0014-4886(74)90118-6

Hogan, M. J., Staff, R. T., Bunting, B. P., Murray, A. D., Ahearn, T. S., Deary, I. J., \& Whalley, L. J. (2011). Cerebellar brain volume accounts for variance in cognitive performance in older adults. Cortex, 47(4), 441-450. https://doi.org/http://dx.doi.org/10.1016/j.cortex.2010.01.001

Huron, D. B. (2006). Sweet anticipation: music and the psychology of expectation. MIT Press.

Hutchinson, S., Lee, L. H.-L., Gaab, N., \& Schlaug, G. (2003). Cerebellar volume of musicians. Cerebral Cortex (New York, N.Y. : 1991), 13(9), 943-9.

Iglói, K., Doeller, C. F., Paradis, A.-L., Benchenane, K., Berthoz, A., Burgess, N., \& RondiReig, L. (2014). Interaction Between Hippocampus and Cerebellum Crus I in SequenceBased but not Place-Based Navigation. Cerebral Cortex (New York, N.Y. : 1991). https:// doi.org/10.1093/cercor/bhu132

Imamizu, H., Kuroda, T., Miyauchi, S., Yoshioka, T., \& Kawato, M. (2003). Modular organization of internal models of tools in the human cerebellum. Proceedings of the National Academy of Sciences of the United States of America, 100(9), 5461-6. https:// doi.org/10.1073/pnas.0835746100

Ito, M. (2008). Control of mental activities by internal models in the cerebellum. Nature 
Reviews Neuroscience, 9(4), 304-313. https://doi.org/10.1038/nrn2332

Jackson, P. L., Meltzoff, A. N., \& Decety, J. (2006). Neural circuits involved in imitation and perspective-taking. NeuroImage, 31(1), 429-439.

https://doi.org/http://dx.doi.org/10.1016/j.neuroimage.2005.11.026

James, C. E., Britz, J., Vuilleumier, P., Hauert, C. A., \& Michel, C. M. (2008). Early neuronal responses in right limbic structures mediate harmony incongruity processing in musical experts. NeuroImage, 42(4), 1597-1608.

https://doi.org/10.1016/j.neuroimage.2008.06.025

James, C. E., Oechslin, M. S., Van De Ville, D., Hauert, C. A., Descloux, C., \& Lazeyras, F. (2014). Musical training intensity yields opposite effects on grey matter density in cognitive versus sensorimotor networks. Brain Structure and Function, 219(1), 353366. https://doi.org/10.1007/s00429-013-0504-z

Juhász, Z. (2004). Segmentation of Hungarian Folk Songs Using an Entropy-Based Learning System. Journal of New Music Research, 33(1), 5-15. https://doi.org/10.1076/jnmr.33.1.5.35395

Justus, T. C., \& Bharucha, J. J. (2001). Modularity in musical processing: the automaticity of harmonic priming. Journal of Experimental Psychology: Human Perception and Performance, 27(4), 1000.

Kajihara, T., Verdonschot, R. G., Sparks, J., \& Stewart, L. (2013). Action-perception coupling in violinists. Frontiers in Human Neuroscience, 7(July), 349. https://doi.org/10.3389/fnhum.2013.00349

Keller, P. E. (2008). Joint Action in Music Performance. Enacting Intersubjectivity: A Cognitive and Social Perspective on the Study of Interactions, 205-221.

Keren-Happuch, E., Chen, S.-H. A., Ho, M.-H. R., \& Desmond, J. E. (2014). A meta-analysis 
of cerebellar contributions to higher cognition from PET and fMRI studies. Human Brain Mapping, 35(2), 593-615. https://doi.org/10.1002/hbm.22194

Kirsch, P., Achenbach, C., Kirsch, M., Heinzmann, M., Schienle, A., \& Vaitl, D. (2003). Cerebellar and hippocampal activation during eyeblink conditioning depends on the experimental paradigm: A MEG Study. Neural Plasticity, 10(4), 291-301. https://doi.org/10.1155/NP.2003.291

Koekkoek, S. K. E., Hulscher, H. C., Dortland, B. R., Hensbroek, R. A., Elgersma, Y., Ruigrok, T. J. H., \& De Zeeuw, C. I. (2003). Cerebellar LTD and Learning-Dependent Timing of Conditioned Eyelid Responses. Science, 301(5640), 1736-1739.

Koelsch, S., Jentschke, S., Sammler, D., \& Mietchen, D. (2007). Untangling syntactic and sensory processing: An ERP study of music perception. Psychophysiology, 44(3), 476490. https://doi.org/10.1111/j.1469-8986.2007.00517.x

Koelsch, S., Schmidt, B.-H., \& Kansok, J. (2002). Effects of musical expertise on the early right anterior negativity: an event-related brain potential study. Psychophysiology, 39(5), 657-663. https://doi.org/10.1017.S0048577202010508

Koeneke, S., Lutz, K., Wüstenberg, T., \& Jäncke, L. (2004). Long-term training affects cerebellar processing in skilled keyboard players. NeuroReport, 15(8).

Koziol, L. F., Budding, D., Andreasen, N., D’Arrigo, S., Bulgheroni, S., Imamizu, H., ... Yamazaki, T. (2014). Consensus paper: The cerebellum's role in movement and cognition. Cerebellum, 13(1), 151-177. https://doi.org/10.1007/s12311-013-0511-x

Krook-Magnuson, E., Szabo, G. G., Armstrong, C., Oijala, M., \& Soltesz, I. (2014). Cerebellar Directed Optogenetic Intervention Inhibits Spontaneous Hippocampal Seizures in a Mouse Model of Temporal Lobe Epilepsy. Eneuro, 1(1).

Lahav, A., Saltzman, E., \& Schlaug, G. (2007). Action representation of sound: audiomotor 
recognition network while listening to newly acquired actions. The Journal of Neuroscience : The Official Journal of the Society for Neuroscience, 27(2), 308-14. https://doi.org/10.1523/JNEUROSCI.4822-06.2007

Landmann, C., Landi, S. M., Grafton, S. T., \& Della-Maggiore, V. (2011). FMRI supports the sensorimotor theory of motor resonance. PLoS ONE, 6(11), 1-8. https://doi.org/10.1371/ journal.pone.0026859

Leggio, M. G., Chiricozzi, F. R., Clausi, S., Tedesco, A. M., \& Molinari, M. (2011). The neuropsychological profile of cerebellar damage: The sequencing hypothesis. Cortex, 47(1), 137-144. https://doi.org/http://dx.doi.org/10.1016/j.cortex.2009.08.011

Lehmann, A., \& Gruber, H. (2006). Music. In K. Ericsson, N. Charness, P. Feltovich, \& R. Hoffman (Eds.), Cambridge Handbook of Expertise and Expert Performance (pp. 457470). Cambridge: Cambridge University Press.

Leiner, H. C. (2010). Solving the Mystery of the Human Cerebellum. Neuropsychology Review, 20(3), 229-235. https://doi.org/10.1007/s11065-010-9140-z

Leiner, H. C., Leiner, A. L., \& Dow, R. S., (1993). Cognitive and language functions of the human cerebellum. Trends Neurosci. 16(11):444-7. https://doi.org/10.1016/0166-

\section{$\underline{2236(93) 90072-\mathrm{T}}$}

Liu, W., Zhang, Y., Yuan, W., Wang, J., \& Li, S. (2012). A Direct Hippocampo-Cerebellar Projection in Chicken. The Anatomical Record: Advances in Integrative Anatomy and Evolutionary Biology, 295(8), 1311-1320. https://doi.org/10.1002/ar.22515

Logan, C. G., \& Grafton, S. T. (1995). Functional anatomy of human eyeblink conditioning determined with regional cerebral glucose metabolism and positron-emission tomography. Proceedings of the National Academy of Sciences of the United States of America, 92(16), 7500-7504. 
Lotze, M., Scheler, G., Tan, H. R. M., Braun, C., \& Birbaumer, N. (2003). The musician's brain: Functional imaging of amateurs and professionals during performance and imagery. NeuroImage, 20(3), 1817-1829. https://doi.org/10.1016/j.neuroimage.2003.07.018

Low, J. K., Ng, H. T., \& Guo, W. (2005). A maximum entropy approach to Chinese word segmentation. In Proceedings of the Fourth SIGHAN Workshop on Chinese Language Processing.

Mackay, D. J. C. (2003). Information Theory, Inference, and Leaning algorithms.

Maidhof, C., Vavatzanidis, N., Prinz, W., Rieger, M., \& Koelsch, S. (2010). Processing expectancy violations during music performance and perception: an ERP study. Journal of Cognitive Neuroscience, 22(10), 2401-2413.

Meyer, L. (1956). Emotion and Meaning in Music. Chicago, IL: University of Chicago Press.

McCallum, A., Freitag, D., \& Pereira, F. C. N. (2000). Maximum Entropy Markov Models for Information Extraction and Segmentation. In Icml (Vol. 17, pp. 591-598).

Musacchia, G., Sams, M., Skoe, E., \& Kraus, N. (2007). Musicians have enhanced subcortical auditory and audiovisual processing of speech and music. Proceedings of the National Academy of Sciences of the United States of America, 104(40), 15894-15898. https://doi.org/10.1073/pnas.0701498104

Narmour, E. (1990). The Analysis and Cognition of Basic Melodic Structures: The Implication-Realization Model. Chicago, IL: Chicago University Press. https://doi.org/10.2307/40285609

O’Reilly, J. X., Beckmann, C. F., Tomassini, V., Ramnani, N., \& Johansen-Berg, H. (2010). 
Distinct and Overlapping Functional Zones in the Cerebellum Defined by Resting State Functional Connectivity. Cerebral Cortex, 20(4), 953-965.

https://doi.org/10.1093/cercor/bhp157

Oechslin, M. S., Van De Ville, D., Lazeyras, F., Hauert, C. A., \& James, C. E. (2013).

Degree of musical expertise modulates higher order brain functioning. Cerebral Cortex, 23(9), 2213-2224. https://doi.org/10.1093/cercor/bhs206

Oganesian, E. A., Melik-Musian, A. B., Fanardzhian, V. V, \& Grigorian, I. (1980). [Morphofunctional analysis of the nature of cerebello-hippocampal connections]. Fiziologicheskii zhurnal SSSR imeni I. M. Sechenova, 66(11), 1632-1639.

Onuki, Y., Van Someren, E. J. W., De Zeeuw, C. I., \& Van der Werf, Y. D. (2015). Hippocampal-Cerebellar Interaction During Spatio-Temporal Prediction. Cerebral Cortex (New York, N.Y. : 1991), 2, 313-321. https://doi.org/10.1093/cercor/bht221

Paleja, M., Girard, T. A., Herdman, K. A., \& Christensen, B. K. (2014). Two distinct neural networks functionally connected to the human hippocampus during pattern separation tasks. Brain and Cognition, 92, 101-111.

https://doi.org/http://dx.doi.org/10.1016/j.bandc.2014.10.009

Pearce, M. T., \& Wiggins, G. A. (2006). The information dynamics of melodic boundary detection. In Proceedings of the Ninth International Conference on Music Perception and Cognition (pp. 860-865).

Pearce, M. T., Müllensiefen, D., \& Wiggins, G. A. (2010). The role of expectation and probabilistic learning in auditory boundary perception: A model comparison. Perception, 39(10), 1367-1391. https://doi.org/10.1068/p6507

Pearce, M. T., Ruiz, M. H., Kapasi, S., Wiggins, G. A, \& Bhattacharya, J. (2010). 
Unsupervised statistical learning underpins computational, behavioural, and neural manifestations of musical expectation. NeuroImage, 50(1), 302-13.

https://doi.org/10.1016/j.neuroimage.2009.12.019

Pearce, M. T., \& Wiggins, G. a. (2012). Auditory Expectation: The Information Dynamics of Music Perception and Cognition. Topics in Cognitive Science, 4(4), 625-652. https://doi.org/10.1111/j.1756-8765.2012.01214.x

Pecenka, N., \& Keller, P. (2009). The relationship between auditory imagery and musical synchronization abilities in musicians. Proceedings of the 7th Triennial Conference of European Society for the Cognitive Sciences of Music (ESCOM 2009), (Escom), 409415.

Peng, H., Zheng, H., Ning, Y., Zhang, Y., Shan, B., Zhang, L., .. Li, L. (2013). Abnormalities of cortical-limbic-cerebellar white matter networks may contribute to treatment-resistant depression: a diffusion tensor imaging study. BMC Psychiatry, 13, 72. https://doi.org/10.1186/1471-244X-13-72

Petrosini, L., Leggio, M. G., \& Molinari, M. (1998). The cerebellum in the spatial problem solving: a co-star or a guest star? Progress in Neurobiology, 56(2), 191-210. https://doi.org/http://dx.doi.org/10.1016/S0301-0082(98)00036-7

Pezzulo, G., Candidi, M., Dindo, H., \& Barca, L. (2013). Action simulation in the human brain: Twelve questions. New Ideas in Psychology, 31(3), 270-290. https://doi.org/10.1016/j.newideapsych.2013.01.004

Phillips, J. R., Hewedi, D. H., Eissa, A. M., \& Moustafa, A. A. (2015). The Cerebellum and Psychiatric Disorders. Frontiers in Public Health, 3, 66. https://doi.org/10.3389/fpubh.2015.00066

Pyper, B. J., \& Peterman, R. M. (1998). Comparison of methods to account for 
autocorrelation in correlation analyses of fish data, 2140, 2127-2140.

https://doi.org/10.1139/f98-104

Ramnani, N., \& Miall, R. C. (2004). A system in the human brain for predicting the actions of others. Nat Neurosci, 7(1), 85-90. https://doi.org/10.1038/nn1168

Ratnaparkhi, A. (1999). Learning to parse natural language with maximum entropy models. Machine Learning, 34(1-3), 151-175.

Reynar, J. C., \& Ratnaparkhi, A. (1997). A maximum entropy approach to identifying sentence boundaries. In Proceedings of the fifth conference on Applied natural language processing (pp. 16-19). Association for Computational Linguistics. https://doi.org/ $10.3115 / 974557.974561$

Rochefort, C., Arabo, A., André, M., Poucet, B., Save, E., \& Rondi-Reig, L. (2011). Cerebellum Shapes Hippocampal Spatial Code. Science, 334(October), 385-389. https:// doi.org/ 10.1126/science.1207403

Rochefort, C., Lefort, J. M., \& Rondi-Reig, L. (2013). The cerebellum: a new key structure in the navigation system. Frontiers in Neural Circuits, 7(March), 35. https://doi.org/10.3389/fncir.2013.00035

Rohrmeier, M. A., \& Koelsch, S. (2012). Predictive information processing in music cognition. A critical review. International Journal of Psychophysiology, 83(2), 164-175. https://doi.org/10.1016/j.ijpsycho.2011.12.010

Salmi, J., Pallesen, K. J., Neuvonen, T., Brattico, E., Korvenoja, A., Salonen, O., \& Carlson, S. (2010). Cognitive and motor loops of the human cerebro-cerebellar system. Journal of Cognitive Neuroscience, 22(11), 2663-76. https://doi.org/10.1162/jocn.2009.21382

Salmi, Rinne, Koistinen, Salonen, \& Alho. (2009). Brain networks of bottom-up triggered 
and top-down controlled shifting of auditory attention. Brain Research, 1286, 155-64. https://doi.org/10.1016/j.brainres.2009.06.083

Schenker, H. (1935). Der freie Satz. Wien: Universal-Edition.

Schmahmann, J. D., \& Sherman, J. C. (1998). The cerebellar cognitive affective syndrome. Brain, 121(4), 561-579. https://doi.org/10.1093/brain/121.4.561

Schoenberg, A. (1978). Theory of Harmony. London, Boston: Faber and Faber.

Schulte-Rüther, M., Markowitsch, H. J., Fink, G. R., \& Piefke, M. (2007). Mirror Neuron and Theory of Mind Mechanisms Involved in Face-to-Face Interactions: A Functional Magnetic Resonance Imaging Approach to Empathy. Journal of Cognitive Neuroscience, 19(8), 1354-1372. https://doi.org/10.1162/jocn.2007.19.8.1354

Schulz, M., Ross, B., \& Pantev, C. (2003). Evidence for training-induced crossmodal reorganization of cortical functions in trumpet players. Neuroreport, 14(1), 157-61. https://doi.org/10.1097/01.wnr.0000053061.10406.c7

Sebanz, N., \& Knoblich, G. (2009). Prediction in Joint Action: What, When, and Where. Topics in Cognitive Science, 1(2), 353-367. https://doi.org/10.1111/j.17568765.2009.01024.x

Shannon, C. E. (1951). Prediction and entropy of printed English. Bell System Technical Journal, 30(1), 50-64. https://doi.org/10.1002/j.1538-7305.1951.tb01366.x

Shukla, D. K., Keehn, B., Lincoln, A. J., \& Müller, R.-A. (2010). White Matter Compromise of Callosal and Subcortical Fiber Tracts in Children With Autism Spectrum Disorder: A Diffusion Tensor Imaging Study. Journal of the American Academy of Child \& Adolescent Psychiatry, 49(12), 1269-1278.e2.

https://doi.org/http://dx.doi.org/10.1016/j.jaac.2010.08.018

Silverman, B. (1986). Density estimation for statistics and data analysis. London; New York: 
Chapman and Hall.

Singer, T., Seymour, B., O’Doherty, J., Kaube, H., Dolan, R. J., \& Frith, C. D. (2004).

Empathy for Pain Involves the Affective but not Sensory Components of Pain. Science, 303(5661), 1157-1162. https://doi.org/10.1126/science.1093535

Snider, R. S., \& Maiti, A. (1976). Cerebellar contributions to the papez circuit. Journal of Neuroscience Research, 2(2), 133-146. https://doi.org/10.1002/jnr.490020204

Steinlin, M. (2008). Cerebellar Disorders in Childhood: Cognitive Problems. The Cerebellum, 7(4), 607-610. https://doi.org/10.1007/s12311-008-0083-3

Stewart, L., Henson, R., Kampe, K., Walsh, V., Turner, R., \& Frith, U. (2003). Brain changes after learning to read and play music. NeuroImage, 20(1), 71-83.

https://doi.org/10.1016/S1053-8119(03)00248-9

Stoodley, C. J., \& Schmahmann, J. D. (2009). Functional topography in the human cerebellum: A meta-analysis of neuroimaging studies. NeuroImage, 44(2), 489-501. https://doi.org/10.1016/j.neuroimage.2008.08.039

Stoodley, C. J. (2012). The cerebellum and cognition: evidence from functional imaging studies. Cerebellum. 2012;11(2):352-65. https://doi.org/10.1007/s12311-011-0260-7

Strange, B., Fletcher, P., Henson, R., Friston, K., \& Dolan, R. (1999). Segregating the functions of human hippocampus, 96(March), 4034-4039. https://doi.org/10.1073/pnas.96.7.4034

Tavano, A., \& Borgatti, R. (2010). Evidence for a link among cognition, language and emotion in cerebellar malformations. Cortex, 46(7), 907-918. https://doi.org/http://dx.doi.org/10.1016/j.cortex.2009.07.017

Thompson, R. F., \& Steinmetz, J. E. (2009). The role of the cerebellum in classical conditioning of discrete behavioral responses. Neuroscience, 162(3), 732-755. 
https://doi.org/http://dx.doi.org/10.1016/j.neuroscience.2009.01.041

Tillmann, B., \& Bigand, E. (2010). Musical structure processing after repeated listening:

Schematic expectationsresist veridical expectations. Musicae Scientiae, (Special Issue), 33-47. https://doi.org/10.1177/10298649100140S204

Tzourio-Mazoyer, N., Landeau, B., Papathanassiou, D., Crivello, F., Etard, O., Delcroix, N., ... Joliot, M. (2002). Automated Anatomical Labeling of Activations in SPM Using a Macroscopic Anatomical Parcellation of the MNI MRI Single-Subject Brain, 289, 273289. https://doi.org/10.1006/nimg.2001.0978

Ungerleider, L. G., Doyon, J., \& Karni, A. (2002). Imaging brain plasticity during motor skill learning. Neurobiology of Learning and Memory, 78(3), 553-564.

https://doi.org/10.1006/nlme.2002.4091

Vuust, P., Brattico, E., Glerean, E., Seppänen, M., Pakarinen, S., Tervaniemi, M., \& Näätänen, R. (2011). New fast mismatch negativity paradigm for determining the neural prerequisites for musical ability. Cortex, 47(9), 1091-1098. https://doi.org/http://dx.doi.org/10.1016/j.cortex.2011.04.026

Vuust, P., Brattico, E., Seppänen, M., Näätänen, R., \& Tervaniemi, M. (2012). The sound of music: Differentiating musicians using a fast, musical multi-feature mismatch negativity paradigm. Neuropsychologia, 50(7), 1432-1443. https://doi.org/http://dx.doi.org/10.1016/j.neuropsychologia.2012.02.028

Vuust, P., Ostergaard, L., Pallesen, K. J., Bailey, C., \& Roepstorff, A. (2009). Predictive coding of music - Brain responses to rhythmic incongruity. Cortex, 45(1), 80-92. https:// doi: 10.1016/j.cortex.2008.05.014

Watson, T. C., Koutsikou, S., Apps, R., \& Jones, M. W. (2015). Distributed networks: new outlooks on cerebellar function. https://doi.org/10.3389/978-2-88919-626-5 
Weiss, A. P., Dewitt, I., Goff, D., Ditman, T., \& Heckers, S. (2005). Anterior and posterior hippocampal volumes in schizophrenia. Schizophrenia Research, 73(1), 103-112. https://doi.org/10.1016/j.schres.2004.05.018

Weiss, C., \& Disterhoft, J. F. (2011). Exploring Prefrontal Cortical Memory Mechanisms with Eyeblink Conditioning. Behavioral Neuroscience, 125(3), 318-326. https://doi.org/ $10.1037 / \mathrm{a} 0023520$

Wikgren, J., Nokia, M. S., \& Penttonen, M. (2010). Hippocampo-cerebellar theta band phase synchrony in rabbits. Neuroscience, 165(4), 1538-1545. https://doi.org/http://dx.doi.org/ 10.1016/j.neuroscience.2009.11.044

Wilson, M., \& Knoblich, G. (2005). The Case for Motor Involvement in Perceiving Conspecifics. Psychological Bulletin, 131(3), 460-473. https://doi.org/10.1037/00332909.131.3.460

Wong, P. C. M., Skoe, E., Russo, N. M., Dees, T., \& Kraus, N. (2007). Musical experience shapes human brainstem encoding of linguistic pitch patterns. Nature Neuroscience, 10(4), 420-422. https://doi.org/10.1038/nn1872

Yu, Q. X., Gao, J. F., Wang, J. J., \& Chen, J. (1989). [Hippocampus-cerebellar cortexcerebellar nuclei projection in the rat: electrophysiological and HRP studies]. Sheng li xue bao : [Acta physiologica Sinica], 41(3), 231-240.

Yu, W., \& Krook-Magnuson, E. (2015). Cognitive Collaborations: Bidirectional Functional Connectivity Between the Cerebellum and the Hippocampus. Frontiers in Systems Neuroscience, 9(December), 177. https://doi.org/10.3389/fnsys.2015.00177

Zacks, J. M., Speer, N. K., Swallow, K. M., Braver, T. S., \& Reynolds, J. R. (2007). Event perception: a mind-brain perspective. Psychological Bulletin, 133(2), 273.

Zatorre, R. J., Chen, J. L., \& Penhune, V. B. (2007). When the brain plays music: auditory- 
motor interactions in music perception and production. Nature Reviews Neuroscience, 8(7), 547-558. https://doi.org/10.1038/nrn2152

\section{Author Note}

The authors declare no competing financial interests. Authors want to thank Brigitte Bogert, Marina Kliuchko, Ben Gold, Johanna Nohrström, Taru Numminen-Kontti, Mikko Heimola, Marita Kattelus, Jyrki Mäkelä, Alessio Falco, Anja Thiede, Suvi Heikkilä for the data collection, Emilia Tuovinen for the help with the behavioral data, Jussi Numminen for checking the MRI scans, Anja Thiede and Lauri Parkkonen for their help with the structural MRI analysis. We thank Emily Carlson, who proofread the manuscript. Simulations were done using taito.csc.fi HP super cluster at CSC - IT Center for Science Ltd., administered by the Ministry of Education, Science and Culture of Finland. This work was supported by the Academy of Finland (project numbers 272250 and 274037) and by the Danish National Research Foundation (project number 117). This paper is based on the third article of the main author's article-based Ph.D dissertation https://jyx.jyu.fi/handle/123456789/52606).

Correspondence concerning this article should be addressed to Iballa Burunat, Department of Music, Art, and Culture Studies, University of Jyväskylä, P.O. Box 35, FI-40014 Jyväskylä, Finland. Phone number: +358 (0) 408054305; E-mail address: Iballa.burunat@jyu.fi. 


\section{MUS > NMUS}

\section{Ant HIPP (L)}

\section{Pos HIPP (L)}
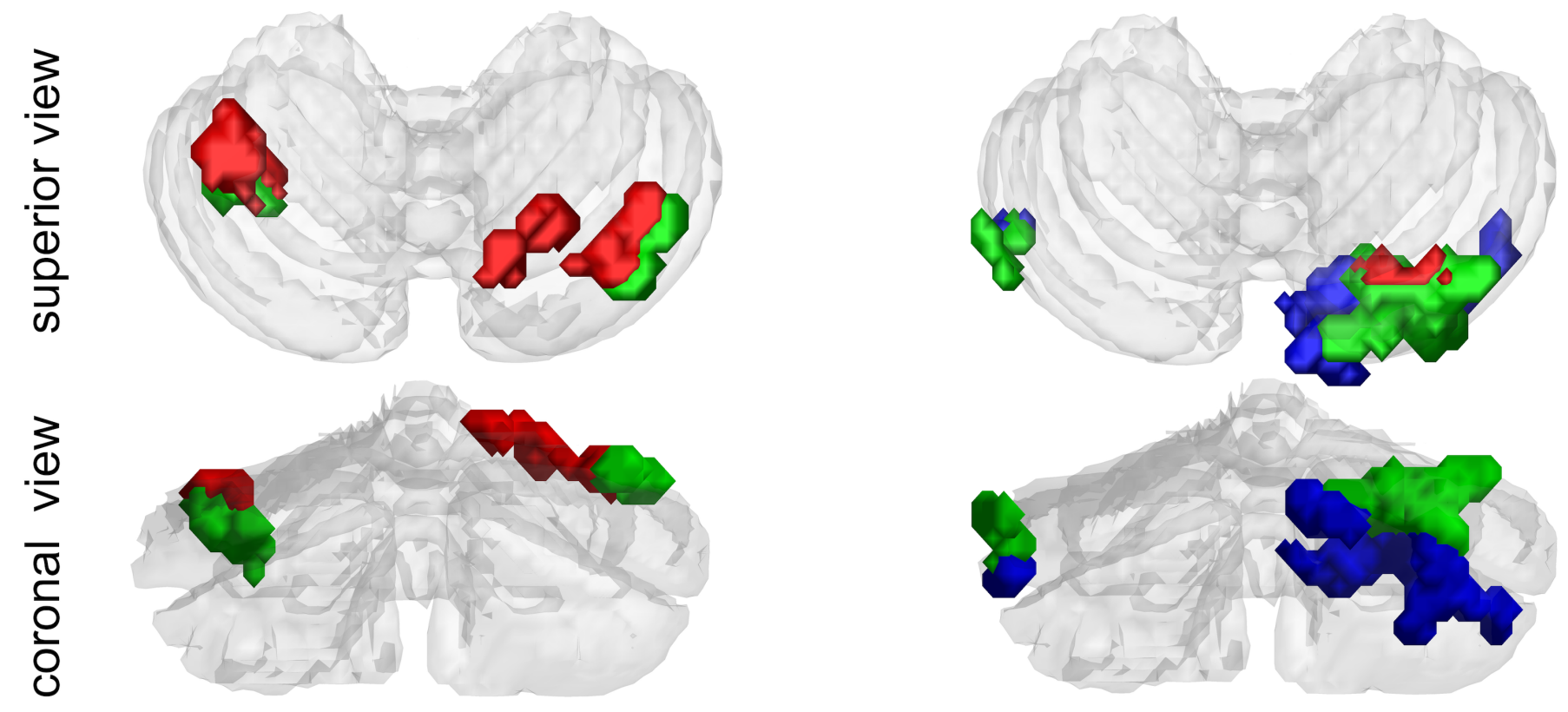

Lob VI Crus I Crus II 


\section{FIGURE CAPTIONS}

Figure 1. Posterior and coronal views of the cerebellum showing regions with increased predictability-driven CER-HIPP functional connectivity in musicians compared to nonmusicians (alpha $=0.01$, one-tailed; cluster-wise threshold $=60$ voxels). Clusters were obtained via the 18 connectivity scheme employed in SPM. Regions encroaching less than 5 voxels were discarded. Abbreviations: Ant: anterior; Pos: posterior; HIPP: hippocampus; L: left; R: right; MUS: musicians; NMUS: nonmusicians. 


\section{Table 1}

Cerebellar regions with increased predictability-driven CER-HIPP functional connectivity in musicians compared to nonmusicians (alpha $=0.01$, one-tailed; cluster-wise threshold $=60$ voxels) and viceversa. Clusters were obtained via the 18 connectivity scheme employed in SPM. Regions encroaching less than 5 voxels were discarded. Abbreviations: k: number of voxels; Ant: anterior; Pos: posterior; HIPP: hippocampus; L: left; R: right; $\max \mathrm{Z}$ : maximal Z-statistic for the region within the cluster; x y z: respective MNI coordinates.

\begin{tabular}{|c|c|c|c|c|c|c|}
\hline \multicolumn{7}{|c|}{ MUSICIANS > NONMUSICIANS } \\
\hline Seed: Ant HIPP (L) & $\mathrm{k}$ & $\max \mathrm{Z}$ & p-value & $\mathrm{x}$ & $\mathrm{y}$ & $\mathrm{Z}$ \\
\hline \multicolumn{7}{|l|}{ Cluster \#1 } \\
\hline Lobule VI (L) & 83 & 3.54 & $p<0.0005$ & -34 & -54 & -26 \\
\hline Crus I (L) & 70 & 3.36 & $p<0.0005$ & -36 & -56 & -26 \\
\hline \multicolumn{7}{|l|}{ Cluster \#2 } \\
\hline Lobule VI (R) & 62 & 3.50 & $p<0.0005$ & 38 & -72 & -20 \\
\hline Crus I (R) & 60 & 3.44 & $p<0.0005$ & 40 & -74 & -20 \\
\hline \multicolumn{7}{|l|}{ Cluster \#3 } \\
\hline Lobule VI (R) & 62 & 3.29 & $p=0.0005$ & 24 & -64 & -16 \\
\hline \multicolumn{7}{|l|}{ Seed: Pos HIPP (L) } \\
\hline \multicolumn{7}{|l|}{ Cluster \#1 } \\
\hline Crus I (R) & 306 & 3.60 & $p<0.0005$ & 32 & -82 & -30 \\
\hline Crus II (R) & 297 & 3.20 & $p<0.001$ & 14 & -76 & -36 \\
\hline Lobule VI (R) & 16 & 2.71 & $p<0.005$ & 24 & -74 & -22 \\
\hline \multicolumn{7}{|l|}{ Cluster \#2 } \\
\hline Crus I (L) & 39 & 3.39 & $p<0.0005$ & -50 & -66 & -28 \\
\hline Crus II (L) & 21 & 2.86 & $p<0.005$ & -44 & -66 & -36 \\
\hline
\end{tabular}




\section{Table 2}

Cerebellar regions showing significant correlation (alpha $=0.05$ one-tailed, uncorrected) between increased predictability-driven connectivity (musicians $>$ nonmusicians) and years of musical training (musicians). Regions encroaching less than 5 voxels were discarded. Abbreviations: k: number of voxels; Ant: anterior; Pos: posterior; HIPP: hippocampus; L: left; R: right; max Z: maximal Z-statistic for the region within the cluster; $\mathrm{x}$ y $\mathrm{z}$ : respective MNI coordinates.

\begin{tabular}{|c|c|c|c|c|c|c|}
\hline Seed: Ant HIPP (L) & $\mathrm{k}$ & $\max Z$ & p-value & $\mathrm{X}$ & $\mathrm{y}$ & Z \\
\hline Lobule VI (R) & 12 & 2.44 & $p<0.01$ & 38 & -68 & -22 \\
\hline Crus I (R) & 6 & 2.20 & $p<0.02$ & 36 & -72 & -22 \\
\hline Lobule VI (R) & 6 & 1.87 & $p<0.05$ & 26 & -64 & -20 \\
\hline
\end{tabular}


Supplementary table S1. Demographic background variables. Table containing the summary results of group comparisons (t-tests or Mann-Whitney U-tests, depending on the normality of the data) for socioeconomic status, cognitive test scores, and personality/mood questionnaire data. There were no significant group differences between musicians and nonmusicians The only variable that came close $(p=0.05)$ was the Stroop color-word naming time; however, when the control Stroop task (color naming) was subtracted from this (which gives a better indication of the Stroop interference effect), it was not even marginally significant any more $(p=0.113)$.

\begin{tabular}{|c|c|c|c|}
\hline & Musicians & Nonmusicians & $p$-value \\
\hline & $N=18$ & $\mathrm{~N}=18$ & \\
\hline \multicolumn{4}{|l|}{ Socioeconomic status (SES) } \\
\hline Hollingshead Index & $44.3 \pm 14.9$ & $34.5 \pm 21.0$ & $0.313(U)$ \\
\hline \multicolumn{4}{|l|}{ Personality } \\
\hline BFQ / Neuroticism scale & $-12.5 \pm 7.2$ & $-9.8 \pm 8.6$ & $0.377(\mathrm{t})$ \\
\hline BFQ / Extroversion scale & $8.0 \pm 11.0$ & $9.4 \pm 8.8$ & $0.905(U)$ \\
\hline BFQ / Openness scale & $22.4 \pm 7.0$ & $17.7 \pm 8.9$ & $0.116(U)$ \\
\hline BFQ / Agreeableness scale & $17.2 \pm 8.4$ & $13.6 \pm 7.8$ & $0.250(\mathrm{t})$ \\
\hline BFQ / Conscientiousness scale & $9.6 \pm 8.6$ & $11.7 \pm 9.7$ & $0.557(\mathrm{t})$ \\
\hline BIS/BAS / Drive scale & $12.5 \pm 3.6$ & $13.4 \pm 2.1$ & $0.448(\mathrm{t})$ \\
\hline BIS/BAS / Fun seeking scale & $14.7 \pm 3.7$ & $14.9 \pm 2.2$ & $0.583(U)$ \\
\hline BIS/BAS / Reward responsiveness scale & $18.5 \pm 3.8$ & $19.1 \pm 3.0$ & $0.651(\mathrm{t})$ \\
\hline BIS/BAS / Inhibition scale & $22.9 \pm 4.3$ & $22.6 \pm 5.4$ & $0.854(\mathrm{t})$ \\
\hline IRI / Fantasy scale & $19.5 \pm 8.1$ & $14.6 \pm 5.2$ & $0.123(\mathrm{t})$ \\
\hline IRI / Perspective taking scale & $19.3 \pm 3.7$ & $17.7 \pm 2.6$ & $0.279(\mathrm{t})$ \\
\hline IRI / Empathic concern scale & $20.6 \pm 3.9$ & $17.8 \pm 5.8$ & $0.200(\mathrm{t})$ \\
\hline IRI / Personal distress scale & $13.2 \pm 7.0$ & $11.2 \pm 5.5$ & $0.479(\mathrm{t})$ \\
\hline \multicolumn{4}{|l|}{ Mood } \\
\hline MADRS / Total score & $4.9 \pm 3.6$ & $5.6 \pm 5.5$ & $0.693(\mathrm{t})$ \\
\hline HADS / Anxiety scale & $3.9 \pm 2.1$ & $5.2 \pm 3.2$ & $0.232(\mathrm{t})$ \\
\hline
\end{tabular}




\begin{tabular}{|l|l|l|l|}
\hline POMS / Total score & $38.8 \pm 14.8$ & $38.4 \pm 15.5$ & 0.941 ( $\mathrm{t})$ \\
\hline Cognitive function & & & \\
\hline WAIS-III / Verbal comprehension index & $115.5 \pm 6.0$ & $113.8 \pm 8.2$ & $0.535(\mathrm{t})$ \\
\hline WAIS-III / Perceptual organization index & $119.0 \pm 9.3$ & $116.5 \pm 8.7$ & $0.473(\mathrm{t})$ \\
\hline WAIS-III / Processing speed index & $116.3 \pm 13.8$ & $115.7 \pm 8.8$ & $0.896(\mathrm{t})$ \\
\hline WMS-III / Working memory index & $108.3 \pm 12.5$ & $110.5 \pm 9.5$ & $0.604(\mathrm{t})$ \\
\hline WMS-III / Word Lists I (immediate recall, standardized score) & $10.4 \pm 2.9$ & $8.8 \pm 3.1$ & $0.184(\mathrm{t})$ \\
\hline WMS-III / Word Lists II (delayed recall, standardized score) & $11.3 \pm 2.9$ & $10.9 \pm 2.6$ & 0.681 (t) \\
\hline TMT / A time (sec) & $24.3 \pm 8.1$ & $26.1 \pm 9.6$ & $0.605(\mathrm{t})$ \\
\hline TMT / B time (sec) & $49.3 \pm 18.8$ & $50.3 \pm 10.7$ & 0.869 (t) \\
\hline TMT / B-A time difference (sec) & $25.0 \pm 15.0$ & $24.2 \pm 7.8$ & 0.865 (t) \\
\hline Stroop / Color naming time (sec) & $62.1 \pm 15.0$ & $67.2 \pm 14.4$ & $0.185(\mathrm{U})$ \\
\hline Stroop / Color-Word naming time (sec) & $93.5 \pm 27.6$ & $105.9 \pm 23.3$ & $0.050(\mathrm{U})$ \\
\hline Stroop / time difference (interference) (sec) & $34.6 \pm 23.6$ & $38.6 \pm 16.3$ & $0.113(\mathrm{U})$ \\
\hline
\end{tabular}

Abbreviations:

$\mathrm{t}=$ Independent samples $\mathrm{t}$-test

$\mathrm{U}=$ Mann-Whitney $\mathrm{U}$ test

BFQ: Big Five Questionnaire

BIS/BAS: Behavioral Inhibition System / Behavioral Approach System

HADS: Hospital Anxiety and Depression Scale

IRI: Interpersonal Reactivity Index

MADRS: Montgomery-Åsberg Depression Rating Scale

POMS: Profile of Mood States

WAIS-III: Wechsler Adult Intelligence Scale III

WMS-III: Wechsler Memory Scale III

TMT: Trail Making Test 